\title{
Estudio descriptivo de las fracturas del radio distal del adulto en España
}

\author{
J. A. Oteo Maldonado', J. Baena ${ }^{2}$, P. Benavente \\ I Servicio de Cirugía Ortopédica y Traumatología. Hospital Universitario de Fuenlabrada. Madrid. España. \\ ${ }^{2}$ Servicio de Cirugía Ortopedica y Traumatología. Hospital FREMAP. Barcelona. España.
}

\begin{abstract}
Resumen: Objetivo: Conocer cual es el estado actual del tratamiento que reciben las fracturas de radio distal del adulto en España.

Material y método: El diseño clínico es un estudio descriptivo. Se recogieron los datos de 279 fracturas de radio distal de 14 centros hospitalarios españoles. Las variables que se tomaron son: edad, sexo, lateralidad, dominancia, grado de actividad física, nivel de energía del accidente, tipo de fractura según la clasificación $\mathrm{AO}$, tipo de tratamiento recibido (ortopédico o quirúrgico) y en los casos cuando se realizó cirugía, tipo de intervención realizada, tiempo transcurrido desde el accidente hasta la realización de la operación quirúrgica, empleo de sustituto óseo, uso de artroscopia y lesión e intervención en lado cubital. Para el estudio estadístico se utilizaron el test de comparación de Chi cuadrado para variables categóricas y el test no paramétrico de Kruskal-Wallis tanto para valorar la dependencia entre el tiempo transcurrido hasta la cirugía y el tipo de fractura, así como entre el tiempo transcurrido hasta la cirugía y el tipo de tratamiento.

Resultados: En 209 casos (74.9\%) el tratamiento fue ortopédico, y en 70 (25.1\%) quirúrgico, de ellos 42 casos se trataron con placa volar, 25 con agujas percutáneas, y 3 con fijador externo. Las fracturas tipo A de la clasificación AO recibieron más tratamiento conservador mientras que las tipo $C$ más tratamiento quirúrgico, dentro del tratamiento quirúrgico las tipo $C$ fueron aquellas donde se realizó una cirugía más compleja (placa volar), mientras que las tipo A y B fueron donde se utilizó fijación con agujas percutáneas. El tiempo de espera para colocar agujas fue más corto que cuando se puso una placa.

Conclusiones: El tratamiento de las fracturas de radio distal fue mayormente ortopédico, el quirúrgico se realizó especialmente en las fracturas complejas. La síntesis con placas se usó sobre todo en fracturas más conminutas (tipo C), mientras que en las fracturas más simples (tipo A y B) se empleó más la síntesis con agujas.
\end{abstract}

Palabras clave: fractura radio distal, placa volar, síntesis de fracturas.

\begin{abstract}
Objective :To determine the current status in treatment of distal radial fractures in Spain, in population over 18 years old.

Methods: Descriptive study. We collected data of 279 fractures in 14 Centers nationwide. The The variables studied were: that are taken are : age, sex, side (left/right), dominance, physical activity level, accident energy level, fracture type (AO classification), treatment (orthopedic/surgical) and in surgical cases, type of intervention, time from accident to surgery, bone substitute use, arthroscopy use, and ulnar side state .In statistical analysis test Chi square was used for categorical variables and nonparametric Kruskal-Wallis test was used for Chi square test was used to analize the categorical variables dependence study between of time to surgery and type of fracture and time to surgery and type of treatment.

Results : 209 cases $(74.9 \%)$ underwent orthopedic treatment , and 70 (25.1 \%) surgical, of which 42 cases were volar plate , $25 \mathrm{~K}$-wire and 3 external fixator. Treatment in type $\mathrm{A}$ are more conservative and in type $\mathrm{C}$ are more surgical. Volar plate was used in type $C$ surgical treatment, and $\mathrm{K}$-wire was used in types $\mathrm{A}-\mathrm{B}$. The waiting time for $\mathrm{K}$-wire surgery was shorter than for plate surgery.

Conclusions : Treatment of distal radius fractures is mostly orthopedic, the surgery was performed particularly in complex fracture. Volar plate was used in type $C$ and $\mathrm{K}$-wires had a role in treatment of type $\mathrm{A}$ and $\mathrm{B}$.
\end{abstract}

Key words: distal radius fracture, volar plate , fracture fixation.

Las fracturas de radio distal pueden llegar a representar un 15\% de todas las fracturas tratadas en urgencias $^{1,2}$. Con el incremento en edad y esperanza de vida de la población se espera que el número absoluto de las mismas se incremente un 50\% en 2030 en los países industrializados ${ }^{3}$, aunque si se observan las tasas de incidencia estandarizadas por edad, hay una tendencia a la disminución en su número, posiblemente derivada de la obesidad presente en la sociedad occidental y del empleo de terapias contra la osteoporosis ${ }^{4}$.
Para el tratamiento de las mismas se emplean distintos métodos que van desde la reducción e inmovilización con yeso, a la osteosíntesis con agujas, fijador exter-

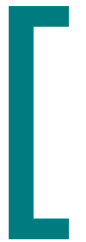


no, clavos intramedulares o diversos sistemas de placas más tornillos, entre los que destaca el uso de placas volares de ángulo fijo ${ }^{5-12}$. A la vez, la artroscopia ${ }^{13,14}$ se ha desarrollado como una herramienta para conseguir mejores resultados en estos pacientes.

Recientes estudios realizados en Estados Unidos muestran un cambio desde el tratamiento conservador hacia la fijación interna en el manejo de estas lesiones, pero son datos procedentes de las compañías de seguros que no cubren al total de la población, y que muestran una variabilidad muy significativa entre regiones $y$ poblaciones de pacientes ${ }^{15,16}$.

Existe poco consenso en relación a las indicaciones para el tratamiento quirúrgico de estas lesiones ${ }^{2}$, en la actualidad la "American Academy of Orthopaedic Surgeos (AAOS)"17 considera que estaría establecida la cirugía en fracturas que tras la reducción presentan un acortamiento radial mayor de $3 \mathrm{~mm}$, inclinación dorsal de la carilla articular del radio mayor de $10^{\circ}$, o un escalón intraarticular mayor de $2 \mathrm{~mm}$, pero el grado de esta recomendación es solo "moderado" dado que el nivel de evidencia sobre el que se apoya es bajo.

El propósito de este artículo es conocer, ante las diferentes opciones que se pueden elegir, cuál es en nuestro medio, el tratamiento que se propone a las fracturas de radio distal en el adulto, y saber en la medida de lo posible qué criterios son en los que basamos esta decisión.

\section{MATERIAL Y MÉTODO}

\section{Población a estudio}

Se propuso, a través de la Comisión de Radio Distal de la Sociedad Española de Cirugía de la Mano (SECMA), la realización de un estudio descriptivo del las fracturas de radio distal en la población adulta española. La difusión del estudio se realizó de tres formas: informando del mismo en las actas de la Sociedad, en la página web de la misma y contactando con un único mensaje con los miembros de la SECMA de forma personal a través del correo electrónico, donde se solicitaba que se recogieran los datos de las fracturas de radio distal vistas en cada centro hospitalario en población adulta durante el periodo del 15 septiembre al 15 de noviembre de 2010 , el correo se acompañaba de un archivo con el cuestionario donde deberían ser recogidos los datos y en el mismo se establecía a quién deberían remitirse dichos resultados.

De todos los cirujanos contactados colaboraron los integrantes de catorce Centros Hospitalarios (- Tabla I. Los casos contribuidos por los diferentes hospitales fueron consecutivos, remitiendo todas las fracturas sucedidas durante el periodo de tiempo establecido. Se recogieron 302 cuestionarios, de los cuales se descartaron 23 que pertenecían a individuos de menos de 18 años de edad, por lo que finalmente se trabajó con los datos de 279 fracturas.

\section{Diseño clínico \\ Estudio descriptivo.}

\section{Instrumentos y medidas}

Edad, sexo, lado, dominancia, grado de actividad (describiendo tres niveles: paciente activo a nivel deportivo y/o profesional, paciente independiente para actividades de la vida diaria aunque ya no activo profesionalmente y paciente dependiente para las actividades de la vida diaria), nivel de energía del accidente (baja energía aquellos que se ocasionaban como consecuencia de una caída desde su propia altura, sin relación con actividad deportiva, ni con accidente de tráfico y de alta energía como los que se producen como consecuencia de una caída de altura o están relacionados con actividad deportiva o accidente de tráfico), tipo de fractura según la clasificación $A O$, tipo de tratamiento recibido (ortopédico o quirúrgico) y en los casos en los que se realizó cirugía, tipo de intervención realizada, tiempo transcurrido desde el accidente hasta la realización de la misma, empleo de sustituto óseo, uso de artroscopia y lesión e intervención en lado cubital.

\section{Análisis estadístico}

Para la recogida y tratamiento inicial de los datos se creó una base de datos en un fichero Excel, en el cual se recodificaron las variables. El análisis estadístico se llevó a cabo con el software SPSS 20.0 (IBM, Chicago, Illinois).

Inicialmente se realizó un estudio descriptivo de los datos, utilizando la distribución de frecuencias para las variables categóricas cualitativas y cuantitativas categorizadas (como la variable "edad", categorizada en quintiles) y la media como medida de tendencia central con la desviación estándar como medida de dispersión para las variables cuantitativas.

Para el estudio estadístico de las variables cualitativas se empleó el test de Chi-cuadrado, recurriendo al test de Fisher cuando más de un 20\% de las casillas presentaban una frecuencia esperada menor a 5 casos en tablas de contingencia de dos por dos o a métodos exactos. 
Para analizar la asociación existente entre el tipo de fractura y el tiempo transcurrido hasta la cirugía, se realizó primero el test de Kolmogorov-Smirnov para el análisis de la normalidad, obteniendo una significación $<0,000$ I, por lo que se rechaza la hipótesis de que la variable sigue una distribución normal. La variable "tipo de fractura" consta de tres categorías ( $\mathrm{A}, \mathrm{B}$ y $\mathrm{C}$ ) uno de los cuales (el tipo $B$ ) es muy pequeño (solo 9 casos), por lo que al tener menos de 10 casos en alguno de los grupos se utilizó la prueba no paramétrica de Kruskal-Wallis.

Esta misma metodología se siguió para estudiar la asociación entre el tipo de tratamiento quirúrgico empleado y el tiempo hasta la cirugía, encontrando en este caso diferencias estadísticamente significativas. Para profundizar y buscar entre qué pareja de grupos estaban realmente dichas diferencias se realizaron comparaciones entre las mismas dos a dos mediante el test de la $U$ de Mann-Whitney, aplicando la corrección de Bonferroni.

\section{RESULTADOS}

De los 279 casos válidos, 213 eran mujeres (76,35\%) y 66 varones $(23,65 \%)$ La edad media fue de 62 años (edad mínima 20, edad máxima 96), estando la mayoría de los pacientes localizados en el rango de edad entre 55 y 80 años (56.27\% del total). Predominó el lado izquierdo frente al derecho (154//25) y en cuanto a la dominancia, la distribución era muy pareja, I 40 no dominante y 139 dominante.

En relación con el grado de actividad se encontró 105 pacientes activos (37.63\%), 142 independientes para sus actividades de la vida diaria (50.89\%) y 32 dependientes ( I I.46\%).

El nivel de energía del accidente fue bajo (caídas desde propia altura) en 253 casos (90.7\%) y alto en 26 pacientes (9.3\%).

El tipo de fractura se recogió según la clasificación $A O$, fue 125 fracturas tipo A (44,8\%), 45 fracturas tipo B $(16,13 \%)$ y 109 fracturas tipo C (39,07\%). Solo hubo un caso en el que la fractura fue abierta.

En relación al tratamiento, en 209 casos (74.9\%) fue ortopédico y 70 casos (25.1\%) se optó por cirugía. Si analizamos el tipo de cirugía realizado, en 42 casos
( I5\%) se utilizó una placa volar, en 25 casos (8,9\%) agujas de kirschner $y$ en 3 casos $(1,07 \%)$ fijador externo.

La media global de espera para la cirugía fue de 8,94 días, con una desviación típica de 10,37 y un intervalo de 0 a 57 días de máximo.

En 3 fracturas (4.22\%) se utilizó sustituto óseo. En 4 casos (5.63\%) se efectuó control por artroscopia.

En $55,94 \%$ de los formularios no estaba relleno el apartado en relación con la situación del cúbito distal y en ninguno de los casos se refirió que se realizara cirugía en la zona.

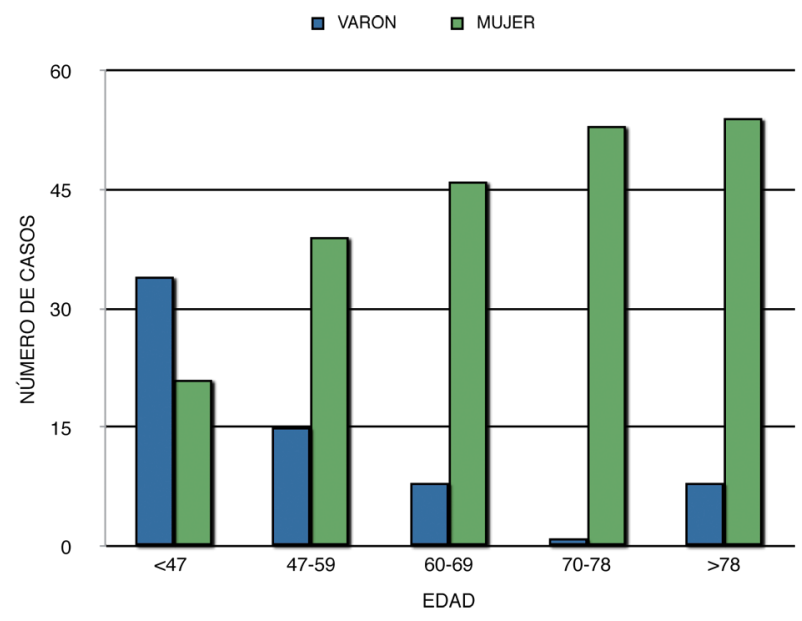

Figura I. Relación sexo y edad.

Al revisar el vínculo entre la edad con el sexo se observó que existía una dependencia estadísticamente significativa entre ambos parámetros \& Figura I y Tabla II.También se apreció esta dependencia entre el tipo de traumatismo y el sexo (-) Tabla III.

No se observó ninguna dependencia entre la edad con el tipo de fractura o el tipo de tratamiento que se realizó sobre las mismas (2) Tabla IV, tampoco entre el sexo con el tipo de fractura o el tipo de tratamiento (2) Tabla $\vee$, ni entre el nivel de actividad con el tipo de fractura o el tipo de tratamiento (- Tabla VI.

Sí que se vio dependencia estadísticamente significativa entre el tipo de fractura y el tipo de tratamiento, según muestran los residuos tipificados corregidos, un mayor número de pacientes con fracturas tipo $A$ siguen un tratamiento conservador, mientras que más fracturas tipo $\mathrm{C}$ se trataron de forma quirúrgica (- Tabla VII. 


\section{TABIA I - HOSPITALES PARTICIPANTES EN EL ESTUdIO}

\begin{tabular}{ccc} 
HOSPITAL & NARCELONA & N DE CASOS APORTADOS \\
HOSPITAL CLÍNIC & & 54 \\
\hline HOSPITAL L'ESPERIT SANT & 7 \\
\hline HOSPITAL FREMAP BARCELONA & 9 \\
\hline HOSPITAL MUTÚA TERRASA & 18 \\
\hline HOSPITAL DE SAN CELONI & 14 \\
\hline HOSPITAL DE TERRASA & 17 \\
\hline HOSPITAL DE VALDEPEÑAS & CIUDAD REAL & 21 \\
\hline FUNDACIÓ SALUT EMPORDÁ & GIRONA & 10 \\
\hline HOSPITAL COMARCAL DE BLANES & \multicolumn{2}{c}{20} \\
\hline HOSPITAL INFANTA CRISTINA & MADRID & 15 \\
\hline HOSPITAL UNIVERSITARIO DE FUENLABRADA & & 18 \\
\hline HOSPITAL COSTA DEL SOL & MÁLAGA & 52 \\
\hline HOSPITAL FREMAP SEVILLA & & 2 \\
\hline
\end{tabular}

Hospitales participantes en el estudio agrupados por provincias.

\section{TABLA \| - Distribución Y RELACIÓn ESTADÍSTICA DE LA EDAD CON EL SEXO.}

\begin{tabular}{|c|c|c|c|c|c|c|c|c|}
\hline & & \multicolumn{6}{|c|}{ GRUPOS DE EDAD } & \multirow[b]{2}{*}{ pt } \\
\hline & & $<47$ & $47-59$ & $60-69$ & $70-78$ & $>78$ & TOTAL & \\
\hline \multirow{3}{*}{ SEXO } & VARÓN & $\begin{array}{c}34 \\
51,51 \%\end{array}$ & $\begin{array}{c}15 \\
22,76 \%\end{array}$ & $\begin{array}{c}8 \\
12,12 \%\end{array}$ & $\begin{array}{c}1 \\
1,51 \%\end{array}$ & $\begin{array}{c}8 \\
12,12 \%\end{array}$ & $\begin{array}{c}66 \\
23,7 \%\end{array}$ & \multirow{2}{*}{0,000} \\
\hline & MUJER & $\begin{array}{c}21 \\
9,85 \%\end{array}$ & $\begin{array}{c}39 \\
13,3 \%\end{array}$ & $\begin{array}{c}46 \\
21,59 \%\end{array}$ & $\begin{array}{c}53 \\
24,88 \%\end{array}$ & $\begin{array}{c}54 \\
25,35 \%\end{array}$ & $\begin{array}{c}213 \\
76,3 \%\end{array}$ & \\
\hline & TOTAL & $\begin{array}{c}55 \\
19,71 \%\end{array}$ & $\begin{array}{c}54 \\
19,35 \%\end{array}$ & $\begin{array}{c}54 \\
19,35 \%\end{array}$ & $\begin{array}{c}54 \\
19,35 \%\end{array}$ & $\begin{array}{c}62 \\
22,22 \%\end{array}$ & $\begin{array}{c}279 \\
100 \%\end{array}$ & \\
\hline
\end{tabular}

En cada casilla se muestra el número de casos y el porcentaje.

† Prueba de Chi Cuadrado. Existe dependencia entre ambas variables

\section{TABLA III - DisTRIBUCIÓN Y RELACIÓN ESTADÍSTICA ENTRE TIPO DE TRAUMATISMO Y SEXO.}

\begin{tabular}{|c|c|c|c|c|c|}
\hline & \multicolumn{2}{|c|}{ TIPO DE TRAUMATISMO } & \multirow[b]{2}{*}{ TOTAL } & \multirow[b]{2}{*}{ p† } \\
\hline & & $\begin{array}{c}\text { BAJA } \\
\text { ENERGÍA }\end{array}$ & $\begin{array}{c}\text { ALTA } \\
\text { ENERGÍA }\end{array}$ & & \\
\hline \multirow{2}{*}{ SEXO } & VARÓN & $\begin{array}{c}47 \\
71,2 \% \\
-6,2 \\
\end{array}$ & $\begin{array}{c}19 \\
28,8 \% \\
6,2 \\
\end{array}$ & $\begin{array}{c}66 \\
23,7 \%\end{array}$ & \multirow{3}{*}{0,001} \\
\hline & MUJER & $\begin{array}{c}206 \\
96,7 \% \\
6,2 \\
\end{array}$ & $\begin{array}{c}7 \\
3,3 \% \\
-6,2 \\
\end{array}$ & $\begin{array}{c}213 \\
76,3 \%\end{array}$ & \\
\hline & TOTAL & $\begin{array}{c}253 \\
90,7 \%\end{array}$ & $\begin{array}{c}26 \\
9,3 \%\end{array}$ & $\begin{array}{c}279 \\
100 \%\end{array}$ & \\
\hline
\end{tabular}

En cada casilla se muestra el número de casos y el porcentaje. † Prueba de Chi Cuadrado. Existe dependencia entre las variables. p 0,001
Al analizar el tiempo transcurrido hasta la cirugía con el tipo de fractura (a) Tabla VIII no se observó dependencia entre el tipo de fractura y el tiempo que pasó hasta que se intervino al paciente.

Por el contrario, sí que se apreció dependencia entre tiempo transcurrido hasta la cirugía y el tipo de tratamiento quirúrgico (2) Tabla IX. Al comparar entre los tres tipos de tratamiento quirúrgico dos a dos, se comprobó que solo había diferencias estadísticamente significativas entre el grupo de agujas y el de placas (4) Tabla X.

Para finalizar, también se apreció dependencia entre el tipo de fractura y el tipo de tratamiento quirúrgico re- 
TAABLA IV - DistRIBUCIÓN Y RELACIÓN ESTADíSTICA DE LA EDAD CON EL TIPO DE FRACTURA Y EL TIPO DE TRATAMIENTO. EN AMBOS CASOS NO EXISTE DEPENDENCIA ENTRE LAS VARIABLES.

\begin{tabular}{|c|c|c|c|c|c|c|c|c|}
\hline & \multicolumn{5}{|c|}{ GRUPOS DE EDAD } & \multirow[b]{2}{*}{ TOTAL } & \multirow[b]{2}{*}{ pt } \\
\hline & & $<47$ & $47-59$ & $60-69$ & $70-78$ & $>78$ & & \\
\hline \multirow{3}{*}{$\begin{array}{c}\text { TIPO } \\
\text { DE FRACTURA }\end{array}$} & $\mathbf{A}$ & $\begin{array}{c}21 \\
16,8 \%\end{array}$ & $\begin{array}{c}24 \\
19,2 \%\end{array}$ & $\begin{array}{c}21 \\
16,8 \%\end{array}$ & $\begin{array}{c}27 \\
21,6 \%\end{array}$ & $\begin{array}{c}32 \\
25,6 \%\end{array}$ & $\begin{array}{c}125 \\
44,8 \%\end{array}$ & \multirow[t]{3}{*}{0,061} \\
\hline & B & $\begin{array}{c}16 \\
35,5 \%\end{array}$ & $\begin{array}{c}8 \\
17,7 \%\end{array}$ & $\begin{array}{c}12 \\
26,6 \%\end{array}$ & $\begin{array}{c}5 \\
11,1 \%\end{array}$ & $\begin{array}{c}4 \\
8,8 \%\end{array}$ & $\begin{array}{c}45 \\
16,1 \%\end{array}$ & \\
\hline & C & $\begin{array}{c}18 \\
16,5 \%\end{array}$ & $\begin{array}{c}22 \\
20,1 \%\end{array}$ & $\begin{array}{c}21 \\
19,2 \%\end{array}$ & $\begin{array}{c}22 \\
20,1 \%\end{array}$ & $\begin{array}{c}26 \\
23,8 \%\end{array}$ & $\begin{array}{c}109 \\
39,1 \%\end{array}$ & \\
\hline \multirow{2}{*}{$\begin{array}{c}\text { TIPO DE } \\
\text { TRATAMIENTO }\end{array}$} & ORTOPÉDICO & $\begin{array}{c}35 \\
16,7 \%\end{array}$ & $\begin{array}{c}39 \\
18,6 \%\end{array}$ & $\begin{array}{c}39 \\
18,6 \%\end{array}$ & $\begin{array}{c}44 \\
21 \%\end{array}$ & $\begin{array}{c}52 \\
24,8 \%\end{array}$ & $\begin{array}{c}209 \\
74,9 \%\end{array}$ & \multirow[t]{2}{*}{0,091} \\
\hline & QUIRÚRGICO & $\begin{array}{c}20 \\
28,5 \%\end{array}$ & $\begin{array}{c}15 \\
21,4 \%\end{array}$ & $\begin{array}{c}15 \\
21,4 \%\end{array}$ & $\begin{array}{c}10 \\
14,2 \%\end{array}$ & $\begin{array}{c}10 \\
14,2 \%\end{array}$ & $\begin{array}{c}70 \\
25,1 \%\end{array}$ & \\
\hline & TOTAL & $\begin{array}{c}55 \\
19,71 \%\end{array}$ & $\begin{array}{c}54 \\
19,35 \%\end{array}$ & $\begin{array}{c}54 \\
19,35 \%\end{array}$ & $\begin{array}{c}54 \\
19,35 \%\end{array}$ & $\begin{array}{c}62 \% \\
22,22 \%\end{array}$ & $\begin{array}{c}279 \\
100 \%\end{array}$ & \\
\hline
\end{tabular}

En cada casilla se muestra el número de casos y el porcentaje.

† Prueba de Chi Cuadrado. En ambos casos no existe dependencia entre las variables

\begin{tabular}{|c|c|c|c|c|c|}
\hline & & \multicolumn{2}{|c|}{ SEXO } & \multirow[b]{2}{*}{ TOTAL } & \multirow[b]{2}{*}{ pt } \\
\hline & & VARÓN & MUJER & & \\
\hline \multirow{3}{*}{$\begin{array}{c}\text { TIPO } \\
\text { DE FRACTURA }\end{array}$} & A & $\begin{array}{c}26 \\
20.8 \%\end{array}$ & $\begin{array}{c}99 \\
79,2 \%\end{array}$ & $\begin{array}{c}125 \\
44,8 \%\end{array}$ & \multirow{3}{*}{0,119} \\
\hline & B & $\begin{array}{c}16 \\
35,5 \%\end{array}$ & $\begin{array}{c}29 \\
64,4 \%\end{array}$ & $\begin{array}{c}45 \\
16,1 \%\end{array}$ & \\
\hline & C & $\begin{array}{c}24 \\
22 \%\end{array}$ & $\begin{array}{c}85 \\
77,9 \%\end{array}$ & $\begin{array}{c}109 \\
39,1 \%\end{array}$ & \\
\hline \multirow{2}{*}{$\begin{array}{c}\text { TIPO DE } \\
\text { TRATAMIENTO }\end{array}$} & ORTOPÉDICO & $\begin{array}{c}46 \\
22 \%\end{array}$ & $\begin{array}{c}163 \\
77,9 \%\end{array}$ & $\begin{array}{c}209 \\
74,9 \%\end{array}$ & \multirow{2}{*}{0,264} \\
\hline & QUIRÚRGICO & $\begin{array}{c}20 \\
28,5 \%\end{array}$ & $\begin{array}{c}50 \\
71,4 \%\end{array}$ & $\begin{array}{c}70 \\
25,1 \%\end{array}$ & \\
\hline & TOTAL & $\begin{array}{c}66 \\
23,7 \%\end{array}$ & $\begin{array}{c}213 \\
76,3 \%\end{array}$ & $\begin{array}{c}279 \\
100 \%\end{array}$ & \\
\hline
\end{tabular}

En cada casilla se muestra el número de casos y el porcentaje. † Prueba de Chi Cuadrado. No existe dependencia entre las variables.

cibido (a) Tabla XI, según muestran los residuos tipificados corregidos, las agujas fueron más utilizadas en los pacientes con fracturas de tipo A, mientras que las placas fueron el tratamiento quirúrgico mayoritario en los pacientes intervenidos con fracturas de tipo C.

\section{DISCUSIÓN}

Hemos observado que la mayoría de las fracturas fueron tratadas de forma ortopédica, pero las más complejas eran más proclives a recibir cirugía. De las técnicas quirúrgicas la más usada fue la placa volar de ángulo fijo, sobre todo en las fracturas más conminutas, pero en las fracturas más sencillas se empleó más la estabilización con agujas. También se ha observado, que la cirugía con agujas se hizo mucho más precoz que la cirugía con placa volar de ángulo fijo.

En relación con la epidemiología de las fracturas, nuestros hallazgos han coincidido con la bibliografía previa. Se ha comprobado que son fracturas más frecuentes en mujeres, y que existe una dependencia entre el sexo y la edad, así como entre el sexo y el tipo de fractura. Es decir, las fracturas se producen especialmente en dos grupos de población, en varones jóvenes relacionadas con traumatismo de alta energía y en mujeres ancianas asociadas a caídas desde su propia altura 2,4,18.

También se ha coincidido con estudios anteriores, tanto en la distribución epidemiológica del tipo de fractu$\mathrm{ra}^{19}$, como en el tipo de tratamiento empleado ${ }^{16}$.

En relación con el tratamiento empleado, la revisión realizada por la Cochrane en 2009 no encuentra evidencias para recomendar un tratamiento específico ${ }^{20}$, el debate para decidir entre tratamiento quirúrgico y no quirúrgico se basa en una cuestión básica: ¿el resultado clínico mejora si se consigue una aproximación mayor a la normal anatomía? Mínimas alteraciones en la superficie articular del radio alteran la distribución de cargas y la cinemática de la muñeca, pero aunque el tratamiento exclusivamente ortopédico reporta un 


\section{TABLA VI - DISTRIBUCIÓN Y RELACIÓN ESTADÍSTICA ENTRE EL NIVEL DE ACTIVIDAD Y EL TIPO DE FRACTURA Y EL TIPO DE TRATAMIENTO}

\begin{tabular}{|c|c|c|c|c|c|c|}
\hline & & \multicolumn{3}{|c|}{ NIVEL DE ACTIVIDAD } & \multirow[b]{2}{*}{ TOTAL } & \multirow[b]{2}{*}{$\mathrm{p} t$} \\
\hline & & ACTIVO & INDEPENDIENTE & DEPENDIENTE & & \\
\hline \multirow{3}{*}{$\begin{array}{l}\text { TIPO DE } \\
\text { FRACTURA }\end{array}$} & A & $\begin{array}{c}43 \\
34,4 \%\end{array}$ & $\begin{array}{c}68 \\
54,4 \%\end{array}$ & $\begin{array}{c}14 \\
11.2 \%\end{array}$ & $\begin{array}{c}125 \\
44,8 \%\end{array}$ & \multirow{3}{*}{0,110} \\
\hline & B & $\begin{array}{c}25 \\
55,5 \%\end{array}$ & $\begin{array}{c}16 \\
35,5 \%\end{array}$ & $\begin{array}{c}4 \\
8,8 \%\end{array}$ & $\begin{array}{c}45 \\
16,1 \%\end{array}$ & \\
\hline & C & $\begin{array}{c}37 \\
33,9 \%\end{array}$ & $\begin{array}{c}58 \\
53,2 \%\end{array}$ & $\begin{array}{c}14 \\
12,8 \%\end{array}$ & $\begin{array}{c}109 \\
39,1 \%\end{array}$ & \\
\hline \multirow{3}{*}{$\begin{array}{c}\text { TIPO DE } \\
\text { TRATAMIENTO }\end{array}$} & ORTOPÉDICO & $\begin{array}{c}72 \\
34,4 \% \\
\end{array}$ & $\begin{array}{c}110 \\
52,6 \% \\
\end{array}$ & $\begin{array}{c}27 \\
12,9 \% \\
\end{array}$ & $\begin{array}{c}209 \\
74,9 \% \\
\end{array}$ & \multirow{3}{*}{0,119} \\
\hline & QUIRÚRGICO & $\begin{array}{c}33 \\
47,1 \% \\
\end{array}$ & $\begin{array}{l}32 \% \\
45,7 \\
\end{array}$ & $\begin{array}{c}5 \\
7,1 \% \\
\end{array}$ & $\begin{array}{c}70 \\
25,1 \% \\
\end{array}$ & \\
\hline & TOTAL & $\begin{array}{c}105 \\
37,6 \%\end{array}$ & $\begin{array}{c}142 \\
50,8 \%\end{array}$ & $\begin{array}{c}32 \\
11,4 \%\end{array}$ & & \\
\hline
\end{tabular}

En cada casilla se muestra el número de casos y el porcentaje.

† Prueba de Chi Cuadrado. En ambos casos no existe dependencia entre las variables

TABLA VII - TABLA DE CONTINGENCIA ENTRE EL TIPO DE FRACTURA Y EL TIPO DE TRATAMIENTO.

\begin{tabular}{|c|c|c|c|c|c|c|}
\hline & \multicolumn{3}{|c|}{ TIPO DE FRACTURA } & \multirow[b]{2}{*}{ TOTAL } & \multirow[b]{2}{*}{$p \dagger$} \\
\hline & & $A$ & $B$ & $C$ & & \\
\hline \multirow{2}{*}{$\begin{array}{c}\text { TIPO } \\
\text { DE } \\
\text { TRATAMIENTO }\end{array}$} & ORTOPÉDICO & $\begin{array}{c}109 \\
87,2 \% \\
4,3\end{array}$ & $\begin{array}{c}36 \\
80,0 \% \\
0,9\end{array}$ & $\begin{array}{c}64 \\
58,7 \% \\
-5,0\end{array}$ & $\begin{array}{c}209 \\
74,9 \%\end{array}$ & \multirow{3}{*}{0,000} \\
\hline & QUIRÚRGICO & $\begin{array}{c}16 \\
12,8 \% \\
-4,3\end{array}$ & $\begin{array}{c}9 \\
20,0 \% \\
-0,9\end{array}$ & $\begin{array}{c}45 \\
41,3 \% \\
5,0\end{array}$ & $\begin{array}{c}70 \\
25,1 \%\end{array}$ & \\
\hline \multicolumn{2}{|c|}{ TOTAL } & $\begin{array}{c}125 \\
100,0 \%\end{array}$ & $\begin{array}{c}45 \\
100,0 \%\end{array}$ & $\begin{array}{c}109 \\
100,0 \%\end{array}$ & $\begin{array}{c}279 \\
100,0 \%\end{array}$ & \\
\hline
\end{tabular}

En cada casilla se muestra el número de casos, el porcentaje en columnas y los residuos tipificados corregidos. $=$ Prueba de Chi Cuadrado. Existe dependencia entre ambas variables.

\section{TABLA VIII - DistRIBUCIÓN DEL TIEMPO HASTA LA CIRUGÍA EN FUNCIÓN DEL TIPO DE FRACTURA.}

\begin{tabular}{c|c|c} 
TIPO DE FRACTURA & media \pm DS & $\begin{array}{c}\text { intervalo en días } \\
\text { (mínimo-máximo) }\end{array}$ \\
\hline$A$ & $7,69 \pm 11,09$ & $0-41$ \\
\hline$B$ & $4,56 \pm 6,98$ & $0-21$ \\
\hline$C$ & $10,24 \pm 10,57$ & $0-57$ \\
\hline
\end{tabular}

$=$ Test de Kruskall-Walli. No existe dependencia entre ambas variables. Test Kruskall-walli p 0,103.
25\% de malas consolidaciones y estos cambios ocasionen artrosis, no siempre esta se van a asociar con pobres resultados funcionales al tratarse de una articulación que no es de carga ${ }^{2,21}$.

Con la aparición de las placas volares de ángulo fijo se ha logrado una mejor reducción de las fracturas ${ }^{22}$, por lo que los últimos años se ha visto un incremento en el número de cirugías y en el número de hospitalizaciones en relación con las fracturas de radio distal con el consiguiente gasto económico asociado ${ }^{4}$. Aunque no con ello se consigue siempre un control completo de las molestias ${ }^{23}$.

En nuestro estudio se continúa la tendencia presente desde la pasada década ${ }^{17,20,22,24}$, de tratar de forma quirúrgica las fracturas más complejas, reservando el tratamiento ortopédico para las más sencillas.

Aunque diferentes autores afirman la ausencia de mejoría asociada a la cirugía, se hace más llamativa conforme aumenta la edad del paciente, y por lo tanto la indicación de cirugía disminuiría en la población anciana $a^{4,25,26}$. En nuestro caso, posiblemente debido al tamaño de la muestra, no se han apreciado diferencias entre los grupos de edad. 


\begin{tabular}{c|c|c} 
TABLA IX - DistRIBUCIÓN DEL TIEMPO HASTA \\
LA CIRUGÍA EN FUNCIÓN DEL TIPODE TRATAMIENTO \\
QUIRÚRGICO. \\
$\begin{array}{c}\text { TIPO TRATAMIENTO } \\
\text { QUIRÚRGICO }\end{array}$ & media \pm DS & $\begin{array}{c}\text { intervalo en días } \\
\text { (mínimo-máximo) }\end{array}$ \\
\hline Agujas & $5,16 \pm 7,96$ & $0-27$ \\
\hline Placa volar & $11,29 \pm 11,08$ & $0-57$ \\
\hline Fijador externo & $7,67 \pm 11,59$ & $0-21$ \\
\hline
\end{tabular}

† Test de Kruskall-Wallis. Existe dependencia entre ambas variables.

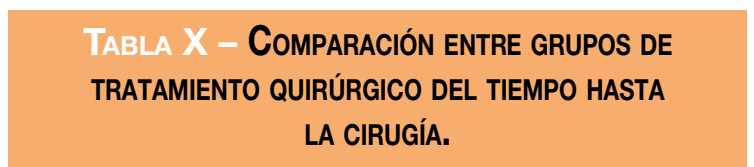

\begin{tabular}{c|c|c}
$\begin{array}{c}\text { TIPO DE TRATAMIENTO } \\
\text { QUIRÚRGICO }\end{array}$ & $p$ & $p$ corregida \\
\hline Agujas vs Placa* & $0,001^{*}$ & $0,003^{*}$ \\
\hline Agujas vs Fijador externo & 0,524 & 1 \\
\hline Placa vs Fijador externo & 0,418 & 1 \\
\hline
\end{tabular}

Comparación por parejas con la $U$ de Mann-Whitney con penalización siguiendo el método de Bonferroni. Existe solo diferencia entres los grupos de agujas y placa.

\section{TABLA XI - TABLA DE CONTINGENCIA ENTRE TIPO DE FRACTURA (CLASIFICACIÓN AO) Y EL TIPO DE TRATAMIENTO QUIRÚRGICO SEGUIDO.}

\begin{tabular}{|c|c|c|c|c|c|c|}
\hline & & \multicolumn{3}{|c|}{ TIPO DE FRACTURA } & \multirow[b]{2}{*}{ TOTAL } & \multirow[b]{2}{*}{$p \dagger$} \\
\hline & & A & B & C & & \\
\hline \multirow{3}{*}{$\begin{array}{l}\text { TIPO TRATAMIENTO } \\
\text { QUIRÚRGICO }\end{array}$} & Agujas & $\begin{array}{c}13 \\
81,2 \% \\
4,3\end{array}$ & $\begin{array}{c}5 \\
55,6 \% \\
1,3\end{array}$ & $\begin{array}{c}7 \\
15,6 \% \\
-4,7\end{array}$ & $\begin{array}{c}25 \\
35,7 \%\end{array}$ & \multirow{4}{*}{0,000} \\
\hline & Placa volar & $\begin{array}{c}3 \\
18,8 \% \\
-3,8\end{array}$ & $\begin{array}{c}4 \\
44,4 \% \\
-1,0\end{array}$ & $\begin{array}{c}35 \\
77,8 \% \\
4,1\end{array}$ & $\begin{array}{c}42 \\
60,0 \%\end{array}$ & \\
\hline & Fijador externo & $\begin{array}{c}0 \\
0,0 \% \\
-1,0\end{array}$ & $\begin{array}{c}0 \\
0,0 \% \\
-0,7\end{array}$ & $\begin{array}{c}3 \\
6,7 \% \\
1,3\end{array}$ & $\begin{array}{c}3 \\
4,3 \%\end{array}$ & \\
\hline \multicolumn{2}{|c|}{ TOTAL } & $\begin{array}{c}16 \\
100,0 \%\end{array}$ & $\begin{array}{c}9 \\
100,0 \%\end{array}$ & $\begin{array}{c}45 \\
100,0 \%\end{array}$ & $\begin{array}{c}70 \\
100,0 \%\end{array}$ & \\
\hline
\end{tabular}

En cada casilla se muestra el número de casos, el porcentaje en columnas y los residuos tipificados corregidos.

$=$ Prueba de Chi Cuadrado. Existe dependencia entre las variables.

Las placas volares de ángulo fijo dan más estabilidad y producen menos complicaciones que las agujas, mientras que estas son baratas, rápidas de poner y, por lo tanto, su tiempo quirúrgico es más corto ${ }^{4,27}$. Por ello, es normal que se recurra a las agujas para la osteosíntesis de las fracturas más sencillas, y que el tiempo de espera para colocarlas sea más corto.

Se ha confirmado la baja incidencia actual en el uso del fijador externo ${ }^{16}$, dado que la fijación interna con placa volar produce mejores resultados funcionales $y$ menos complicaciones ${ }^{28}$. La fijación externa se reserva para situaciones límite como fracturas abiertas o politraumatizados?

Se ha observado una muy baja incidencia en el uso de substitutos óseos, posiblemente debido a la escasa evidencia científica vista en relación con el empleo de estos derivados ${ }^{29}$.

A pesar del incremento en el número de publicaciones que informan de los beneficios del empleo de la artroscopia en el tratamiento de las fracturas de radio distal13,14, la utilización de la misma en nuestra muestra ha sido muy bajo. La necesidad de reorganizar el quirófano, el temor a aumentar el tiempo de cirugía y el costo de la misma, puede que sea lo que retraiga a la gran mayoría de los cirujanos del uso de esta técnica.

Aunque algunos autores han considerado que la importancia del compromiso del cúbito distal en las fracturas de radio distal es evidente ${ }^{30}$, cada vez con más frecuencia aparecen artículos que informan que la presencia o no de fractura en la estiloides cubital no influ- 
ye en el resultado final de la lesión del radio ${ }^{31}$, es por esta razón, por la que se supone que los cirujanos que han participado en la muestra hayan minusvalorado de forma generalizada la información de esta zona.

Para finalizar, indicaremos las limitaciones del estudio. La primera, es no haber conseguido una colaboración de un mayor número de hospitales, que hubiesen proporcionado una información más detallada sobre el problema, el segundo, es desconocer quién trataba en cada centro las fracturas, puesto que se ha visto que en aquellos lugares donde no existen unidades de cirugía de mano, se tiende más al tratamiento ortopédico que donde sí existen estas unidades ${ }^{16}$ y el tercero, es la falta de información acerca de la situación sobre la parte cubital de la muñeca, al haberse dejado sin rellenar más del $50 \%$ de los formularios.

Este estudio es una aportación relevante sobre la situación actual del tratamiento de las fracturas de muñeca, puesto que se trata de un trabajo multicéntrico que abarca diferentes áreas del estado español recogiendo un número elevado de casos. Dada la coyuntura económica actual puede ser considerado como un primer paso a partir del cual desarrollar nuevos estudios, enfocados a definir el coste beneficio de los diferentes tratamientos empleados.

\section{CONCLUSIÓN}

En el momento actual en España el tratamiento que reciben mayoritariamente las fracturas de radio distal es ortopédico, empleándose la cirugía en las fracturas más complejas. Dentro de los diferentes tratamientos quirúrgicos, el más usado es la placa volar que se utiliza en las lesiones con mayor conminución (tipo C), seguido por el empleo de las agujas percutáneas, las cuales continúan teniendo un papel en la síntesis de las fracturas más simples (tipo A y B). Cuando en la cirugía se colocan agujas, esta es mucho más precoz en el tiempo que en el caso de emplearse placas volares.

\section{CONFLICTOS DE INTERESES}

Los autores declaran no tener conflictos de intereses.

\section{BIBLIOGRAFÍA}

I. Lenoble E, Dumontier C. Fracture de l'extremité distal des deux os de l'avant-bras chez l'adulte. En: EMC.
Appareil locomoteur. Paris: Éditions scientifiques et médicales Elsevier; 1997, I4-045-B- 10.

2. Laino DK, Tejwani N. Indications for operative fixation of distal radius fractures: a review of the evidence. Bull NYU Hosp Jt Dis. 20 I2;70( I):35-40,

3. Figl M, Weninger P, Jurkowitsch J, Hofbauer J, Schauer J, Leixnering M. Unstable distal radius fractures in the elderly patient-volar fixed-angle plate osteosynthesis prevents sencodary loss of reduction. J Trauma. 20 10;68(4):992-8.

4. de Putter CE, Selles RW, Polinder S, Hartholt KA, Looman CW, Panneman MJ, et al. Epidemiology and health care utilisation of wrist fractures in older adults in The Netherlands, 1997-2009. Injury. 2012:44(4):421-6.

5. Rayhack JM. The history and evolution of percutaneous pinning of displaced distal radius fractures. Orthop Clin North Am. 1993;24(2):287-300.

6. Böhler J. [Juxta-articular forearm fractures]. Chirurg. 1969;40(5): 198-203.

7. Kennedy SA, Hanel DP. Complex distal radius fractures. Orthop Clin North Am. 20 13;44(I):81-92.

8. Kamath AF, Zurakowski D, Day CS. Low-profile dorsal plating for dorsally angulated distal radius fractures: an outcomes study. J Hand Surg Am. 2006;3 I (7): I 06 I -7.

9. Orbay JL, Fernandez DL. Volar fixation for dorsally displaced fractures of the distal radius: a preliminary report. J Hand Surg Am. 2002;27(2):205- 15.

10. Rausch S, Klos K, Stephan H, Hoffmeier K, Gras F, Windolf $M$, et al. Evaluation of a polyaxial angle-stable volar plate in a distal radius C-fracture model- a biomechanical study. Injury. 20 I I;42(I I): 1248-52.

I I. Saw N, Roberts C, Cutbush K, Hodder M, Couzens G, Ross M. Early experience with the TriMed fragmentspecific fracture fixation system in intraarticular distal radius fractures. J Hand Surg EurVol. 2008;33 (I):53-8.

12. Nishiwaki M, Tazaki K, Shimizu H, llyas AM. Prospective study of distal radial fractures treated with an intramedullary nail. J Bone Joint Surg Am. 201 I;93(I5): 1436-4I.

13. Del Cerro M, De las Heras J, García D, Martín A, Vaquero J. Utilidad de la artroscopia en el tratamiento de las fracturas de la extremidad distal del radio. Pat Ap Locom. 2007:5:64-71.

14. Del Piñal F. Technical tips for (dry) arthroscopic reduction and internal fixation of distal radius fractures. J Hand Surg Am. 20 I I;36( I0): 1694-705.

15. Chung KC, Shauver MJ, Birkmeyer JD. Trends in the United States in the treatment of distal radial fractures in the elderly. J Bone Joint Surgery Am. 2009;9I (8): I868-73

16. Chung KC, Shauver MJ,Yin H, Kim HM, Baser O, Birkmeyer JD.Variations in the use of internal fixation for 
distal radial fracture in the United States Medicare population. J Bone Joint Surg Am. 201 1; 93(23):215462.

17. The treatment of distal radius fractures: guideline and evidence report. Adopted by the American Academy of Orthopaedic Sugeons Board of Directors, decembrer 5, 2009. Rosemont, IL: America Academy of Orthopaedic Surgeons; 2009.

18. Choueka J, Rubio F. Distal radius fractures: overview and algorithms for treatment. En:Trumble TE, Budoff JE (Ed). Hand Surgery Update IV. Rosemont, IL: American Society for Surgery of the Hand; 2007. p. 75-95.

19. McQueen MM. Epidemiology of fractures of the radius and ulna. En: McQueen MM, Jupiter JB, editors. Radius and ulna. Oxford: Butterworth Heinemann; 1999.

20. Handoll HH, Madhok R. WITHDRAWN: Surgical interventions for treating distal radial fractures in adults. Cochrane Database Syst Rev. 2009 8;(3):CD003209.

21. McGrory BJ, Amadio PC. Malunion of the distal radius. En: Cooney WP, Linscheid RL, Dobyns JH, editores. The Wrist: Diagnosis and Operative Treatment. St. Louis: Mosby; 1998. p. 356-3.

22. Kiliç A, Kabukçuoğlu Y, Ozkaya U, Gül M, Sökücü $S$, Ozdoğan U. Volar locking plate fixation of unstable distal radius fractures. Acta Orthop Traumatol Turc. 2009:43(4):303-8.

23. Kurimoto $S$, Tabete M, Shinohara T, Arai T, Hirata $H$. Residual wrist pain after volar locking plate fixation of distal radius fractures. Acta Orthop Belg 2012, (5):603- 10.

24. Gehrmann SV, Windolf J, Kaufmann RA. Distal radius fracture management in elderly patients: a literature review. J Hand Surg Am. 2008; 33(3):421-9.
25. Arora R, Lutz M, Deml C, Krappinger D, Haug L, Gabl $M$. A prospective randomized trial comparing nonoperative treatment with volar locking plate fixation for displaced and unstable distal radial fractures in patients sixty-five years of age and older. J Bone Joint Surg Am. 201 I;93(23):2146-53.

26. Diaz-Garcia RJ, Oda T, Shauver MJ, Chung KC. A systematic review of outcomes and complications of treating unstable distal radius fractures in the elderly. J Hand Surg Am. 20 I I; 36(5):824-35.

27. Maire N, Lebailly F, Zemirline A, Hariri A, Facca S, Livemeaux P. Prospective continuous study comparing intrafocal cross-pinnng HK2 (®) with a locking plate in distal radius fracture fixation. Chir Main. 2013 Feb;32(I): 17-24.

28. Xie X, Xie X, Qin H, Shen L, Zhang C. Comparison of internal and external fixation of distal radius fractures. Acta Orthop. 2013 Jun;84(3):286-91.

29 Handoll HH,Watts AC. Bone grafts and bone substitutes for treating distal radial fractures in adults. Cochrane Database Syst Rev. 2008 16;(2):CD006836.

30. Oskarsson GV, Aaser P, Hjall A. Do we underestimate the predictive value of the ulnar styloid affection in Colles fractures? Arch Orthop Trauma Surg. 1997; | I6(6-7):341-4.

3I Souer JS, Ring D, Matschke S, Audige L, Marent-Huber M, Jupiter JB; AOCID Prospective ORIF Distal Radius Study Group. Effect of an unrepaired fracture of the ulnar styloid base on outcome after plate-and-screw fixation of a distal radial fracture. J Bone Joint Surg Am. 2009;9I (4):830-8. 